\title{
A PREVALÊNCIA DE DEPRESSÃO NOS IDOSOS QUE PARTICIPAM DO PROJETO DE EXTENSÃO “QUALIDADE DE VIDA NA TERCEIRA IDADE” NO CRAS VILA NOVA EM GURUPI-TO
}

\author{
THE PREVALENCE OF DEPRESSION IN THE ELDERLY PARTICIPATING IN THE PROJECT OF \\ EXTENSION "QUALITY OF LIFE IN THE THIRD AGE" IN THE NEW CRAS VILA IN GURUPI-TO
}

Maria Elena Alves Coelho Teles ${ }^{1}$, Renata Rodrigues Rocha², Tânia Maria Lago $^{3}$

\section{RESUMO}

A depressão é o transtorno psiquiátrico mais comum entre os idosos, geralmente sem diagnóstico e nem tratamento adequado. As consequências desta doença afetam diretamente a qualidade de vida dos idosos e prejudicam a sua interação e integração social. O presente estudo teve como objetivo verificar a prevalência de depressão em idosos que participam do Projeto de Extensão "Qualidade de Vida na Terceira Idade" no CRAS Vila Nova - Gurupi-To. A pesquisa foi realizada com vinte idosos participantes do referido projeto de extensão, tendo como instrumento de pesquisa o BDI (Inventário de Depressão Beck), que foi aplicado aos sujeitos participantes de forma individual, e as informações coletadas analisadas quantitativamente. Verificouse que a prevalência da depressão nos idosos participantes da pesquisa foi de um total de 9 idosos equivalente a (45\%) apresentaram depressão leve, 8 idosos equivalente a (40\%) apresentaram depressão moderada e 3 idosos equivalente (15\%) apresentaram depressão mínima. Diante dos dados apresentados foi possível averiguar que não houve indicativos de depressão grave nos participantes do Projeto de Extensão Qualidade de Vida na terceira idade do CRAS - Vila Nova, sendo importante o acompanhamento dos casos de depressão moderada para que não evolua para quadros mais graves.

Palavras-chave: Psicologia; Depressão na Terceira Idade; Grupo de Idosos.

\section{ABSTRACT}

The Depression and the psychiatric disorder most common among the elderly, often undiagnosed and treatment Suitable votes. As consequences this disease directly affect the quality of life of the elderly and harm YOUR Interaction and Social Integration. The present study aimed to verify one Depression Prevalence in the Elderly What part of the Extension Project "Quality of Life in Old Age" in CRAS Vila Nova - Gurupi-To. The survey was conducted with XX Senior said participants Extension Project, tendon as search tool BDI (Beck Depression Inventory), which was applied to the subjects individually Participants, and how INFORMATION collected quantitatively analyzed. It was que one Depression Prevalence In Senior Research Participants was um total of 9 Elderly equivalent to (45\%) had mild depression, 8 Elderly equivalent to (40\%) had moderate depression and 3 equivalent Seniors (15\%) Minimum presented Depression. Before the data presented was possible to ascertain que no was indicative of severe depression a Quality Extension Project Participants Life in the Third Age CRAS - Vila, Important BEING The S Monitoring OF Moderate Depression Cases to prevent development of Tables MORE graves.
${ }^{1}$ Graduada em Psicologia pelo Centro Universitário Unirg Gurupi-TO, Especialista em Psicologia Organizacional e do Trabalho

E-mail: elena2007gp@gmail.com

${ }^{2}$ Graduada em Psicologia pelo Centro Universitário Unirg Gurupi-TO.

${ }^{3}$ Prof do curso de Psicologia do Centro Universitário Unirg Gurupi-TO e Doutoranda em Psicologia Investigativa

Keywords: Psychology; Depression in the Elderly ; Group of Elderly. 


\section{INTRODUÇAOO}

Atualmente, o Brasil possui aproximadamente 17 milhões de habitantes com mais de 60 anos, ocupando destaque entre os dez maiores países com populações de idosos. (SILVA; SANTOS, 2010).

Segundo Garbin, et al. (2010) a população idosa representa 9\% no Brasil, totalizando mais de 14,5 milhões de idosos, e as estimativas indicam que até 2020 essa população será de 12\%. Nessa perspectiva estima-se que, no ano de 2025 a população contará com mais de 30 milhões de habitantes com idade acima de sessenta anos.

De acordo com o Estatuto do Idoso (2003):

Art. 1으, é considerado idoso às pessoas com idade igual ou superior a 60 (sessenta) anos.

Art. $2^{\circ}$, os direitos fundamentais inerentes à pessoa humana, sem prejuízo da proteção integral de que trata esta Lei, assegurando-se lhe, por lei ou por outros meios, todas as oportunidades e facilidades, para preservação de sua saúde física e mental e seu aperfeiçoamento moral, intelectual, espiritual e social, em condições de liberdade e dignidade.

Segundo Melo e Teixeira (2011) com o chegar da idade os indivíduos vão perdendo sua vitalidade e suas forças vão diminuindo, o mundo parece não ter mais o espaço que tinha antes. Com a perda das atividades em decorrência do envelhecer, o idoso sente-se cada vez mais dependente e menos ativo, com isso os principais consequências são: redução da rede social, problemas conjugais, perda do poder aquisitivo e a vulnerabilidade às diversas doenças.

$\mathrm{Na}$ contemporaneidade as queixas do público idoso, deixaram de ser apenas físicas e vem apresentando cada vez mais problemas relacionados ao psicossocial entre elas estão à depressão, que é o transtorno psiquiátrico mais comum entre os idosos, geralmente sem diagnóstico e nem tratamento adequado, onde as consequências desta doença afetam diretamente a qualidade de vida do idoso (PARADELA; MELO; TEIXEIRA, 2011).

Segundo Paradela (2011), os principais sintomas da depressão em idosos são: choro frequente, sentimento de tristeza e inutilidade, desesperança, culpa, comportamento de apatia, ideias de morte, falta de energia, dores difusas, alterações no sono, apetite e hábito intestinal e diminuição da libido.

Deve-se ter um olhar especial em idosos com sintomas depressivos, de acordo com a frequência destes e as consequências negativas na qualidade de vida. Tendo em vista que as causas da depressão podem ser fatores psicossociais ou de motivos 
adversos, que podem contribuir para o início da depressão, podendo ter influências genéticas, mas também desencadeada através de acontecimentos cotidianos negativos. Desse modo existem fatores contribuintes da depressão em idosos como: solidão, viuvez, divórcio, baixo nível de instrução, abuso de substâncias como: álcool e tabaco são fatores contribuintes da depressão neste ciclo vital (BORGES; DALMOLIN, 2012).

Diante da literatura apresentada pelos autores Borges e Dalmolin (2012) e os autores Paradela; Melo; Teixeira (2011) sobre depressão em idosos, mostram-se relevantes os tratamentos clínicos, onde as intervenções psicológicas precoces têm mostrado resultados, ressaltando a importância da prevenção dos fatores de risco.

Assim a avaliação do diagnóstico depressivo em idosos tem sido efetivada através de aplicação de escalas reconhecidas mundialmente, pois estas facilitam uma análise nessa faixa etária (SILVA et al., 2012). Na pesquisa foi utilizado o Inventário de depressão de Beck, que segundo Beck e Steer (1993), trata-se de uma escala de autorrelato, para levantamento da intensidade dos sintomas depressivos.

Os tratamentos visam reduzir os sintomas depressivos do idoso, proporcionar apoio emocional, reduzir a ansiedade e intervir diretamente na elevação da confiança e autoestima, com o objetivo de melhorar a qualidade de vida do indivíduo, as intervenções psicológicas que tem se mostrado mais eficazes nesse contexto são: Psicoterapia Breve; Terapia Cognitiva comportamental e Terapia de Grupo terapêutico (CARVALHO \& FERNANDES, 2002; MELLO \&TEIXEIRA, 2011).

Segundo os autores Carvalho e Fernandes (2002) os grupos terapêuticos com idosos depressivos têm mostrado eficácia, pois através destes os idosos veem a oportunidade de compartilhar experiências, angústias, desejos de uma melhor qualidade de vida. Esses grupos terapêuticos com idosos têm a finalidade de socializar os mesmos, proporcionando aumento da autoestima e resolução de conflitos intrapsíquicos e interpessoais.

A estimativa é que as expectativas de vida dos idosos irão aumentar cada vez mais, onde a sociedade deve entender que o envelhecer é um processo de contínuas mudanças, adaptações que merecem cuidados específicos para se ter uma vida digna e saudável (ARAUJO, 2012). Assim núcleos de convivência têm surgido, com o objetivo de melhorar a qualidade de vida dos idosos.

De acordo com o Ministério do Desenvolvimento Social (MDS) é ofertado nos Centros de Referência e Assistência Social (CRAS) grupos de idosos que não possuem acesso a serviços sociais. Em Gurupi-To, o Centro de Referência e Assistência Social 
(CRAS) Vila Nova, existe desde agosto de 2015 e há um trabalho especifico voltado a essa clientela, sendo que em agosto de 2015 o Projeto de Extensão "Qualidade de Vida na Terceira Idade" do Centro Universitário Unirg passou a ser realizado na instituição.

A problemática sugerida foi depressão nos idosos que frequentam o Projeto de Extensão "Qualidade Vida na Terceira Idade" na instituição CRAS Vila Nova visto que esses sintomas afetam diretamente a qualidade de vida dos idosos.

O objetivo do artigo foi pesquisar a prevalência de depressão nos idosos que participam do Projeto de Extensão "Qualidade de Vida na Terceira Idade" no CRAS Vila Nova - Gurupi-To.

\section{MATERIAIS E MÉTODOS}

Foi realizada uma pesquisa de campo, do tipo exploratória por verificar a prevalência de depressão num grupo de idosos, e descritiva por tentar descrever o contexto e razões que resultaram no processo depressivo. O fenômeno estudado foi à depressão em idosos que participam do Projeto de Extensão "Qualidade de Vida na Terceira Idade" no CRAS Vila Nova - Gurupi-To.

Participaram da pesquisa um total de 20 idosos que frequentam assiduamente, há pelo menos 10 meses, o Projeto de Extensão "Qualidade de Vida na Terceira Idade" no CRAS Vila Nova - Gurupi-To. Foram excluídos os idosos que não participam do projeto de extensão em questão e sim de outras atividades desenvolvidas na instituição, e também aqueles que não concordaram em assinar o Termo de Livre Esclarecimento (TCLE).

Após aprovação da pesquisa pelo Comitê de Ética do Centro Universitário Unirg, parecer $n=1.439 .233$, foi dado início a coleta de dados. Foi aplicado um questionário individualmente com cada participante do grupo. Este questionário faz parte das Escalas Beck, sendo chamado BDI (Inventário de depressão Beck) segundo Beck \&Steer (1993), trata-se de uma escala de autor relato, consiste de 21 itens, incluindo sintomas e atitudes, cuja intensidade varia de 0 a 3 . Referem-se à tristeza, pessimismo, sensação de fracasso, falta de satisfação, sensação de culpa, sensação de punição, autodepreciação, autoacusações, ideias suicidas, crises de choro, irritabilidade, retração social, indecisão, distorção da imagem corporal, inibição para o trabalho, distúrbio do sono, fadiga, perda de apetite, perda de peso, preocupação somática, diminuição de libido (CUNHA, 2011). 
Assim, para classificar os níveis de depressão nos idosos que participaram da pesquisa, foram utilizados os seguintes pontos de corte: menor que 0 a $11=$ sem depressão ou depressão mínima; 12 a 19 = depressão leve; de 20 a $35=$ depressão moderada; de 36 a 63 = depressão grave (CUNHA, 2011).

Esse instrumento de pesquisa (BDI) não estará em anexo, pois, conforme a Resolução do Conselho Federal de Psicologia CFP no 002/2003 art. $1^{0}$ "Os testes Psicológicos são instrumentos de avaliação ou mensuração de características psicológicas, constituindo-se um método ou uma técnica de uso privativo do psicólogo".

Os dados foram coletados no CRAS Vila Nova na cidade de Gurupi-To que propiciou infraestrutura adequada para realização da pesquisa e bem estar dos sujeitos envolvidos, reduzindo os possíveis riscos aos participantes, caracterizados principalmente por desconforto ao responder o BDI. As perguntas do instrumento foram feitas em forma de entrevista considerando aqueles idosos que são analfabetos, para não denegrir sua imagem, física, psíquica, moral, intelectual, social, cultural ou espiritual, conforme Res. CNS no 0 466/2012.

Os dados quantitativos coletados através do BDI foram organizados, tabulados e as frequências de respostas transformadas em valores percentuais.

\section{RESULTADOS E DISCUSSÃO}

Tabela 1 - Itens dos sintomas de depressão do Inventário de Beck - BDI

\begin{tabular}{lcc|lcc}
\hline INDICADORES & SIM & NÃO & INDICADORES & SIM & NÃO \\
\hline Diminuição da libido & $95 \%$ & $5 \%$ & Sensação de punição & $40 \%$ & $60 \%$ \\
\hline Distúrbio do sono & $90 \%$ & $10 \%$ & Distorção da imagem corporal & $40 \%$ & $60 \%$ \\
\hline Fadiga & $85 \%$ & $15 \%$ & Crises de choro & $35 \%$ & $65 \%$ \\
\hline Inibição para trabalho & $80 \%$ & $20 \%$ & Perda de peso & $30 \%$ & $70 \%$ \\
\hline Tristeza & $70 \%$ & $30 \%$ & Retração social & $25 \%$ & $75 \%$ \\
\hline Preocupação somática & $65 \%$ & $35 \%$ & Sensação e fracasso & $25 \%$ & $75 \%$ \\
\hline Irritabilidade & $60 \%$ & $40 \%$ & Sensação de culpa & $25 \%$ & $75 \%$ \\
\hline Falta de satisfação & $55 \%$ & $45 \%$ & Autoacusações & $20 \%$ & $80 \%$ \\
\hline Perda de apetite & $50 \%$ & $50 \%$ & Autopreciação & $10 \%$ & $90 \%$ \\
\hline Indecisão & $50 \%$ & $50 \%$ & Ideias suicidas & $0 \%$ & $100 \%$ \\
\hline Pessimismo & $20 \%$ & $80 \%$ & & & \\
\hline
\end{tabular}

Conforme a (Tabela 1) foi possível verificar que os indicadores que apresentaram resultados com respostas mais discrepantes entre si foram: diminuição da libido um total de 19 idosos equivalentes a (95\%), e um idoso equivalente a (5\%) não perdeu o interesse por sexo. Com relação ao indicador distúrbio do sono um total de 18 idosos equivalentes a $(90 \%)$ responderam que não conseguem dormir como antes, entretanto um total de 2 
idosos equivalente (10\%) não perceberam nenhuma mudança significativa com relação ao sono.

Foi possível verificar com o indicador fadiga um total de 17 idosos equivalente (85\%) apresentaram este sintoma e 3 idosos (15\%) não apresentaram. Enquanto 0 indicador inibição para o trabalho um total de 16 idosos (80\%) apresentaram déficit e 4 idosos (20\%) tem disposição para trabalhar. O indicador tristeza observou-se que um total de 14 idosos (70\%) sentem este sintoma e 6 idosos equivalente a (30\%) responderam que não sentem.

Segundo o Manual das Escalas de Beck (BDI), deve-se dar atenção especial a avaliação feita no item de pessimismo e ideias suicidas, dada a sua relevância para estimar a existência de potencial suicida (CUNHA, 2011).

A partir da interpretação se faz necessário citar que os resultados com relação aos indicadores que se devem ter atenção especial com a pesquisa, foi possível verificar que dos 20 idosos equivalente a (100\%) não apresentaram ideias suicidas o que demonstra que estes indivíduos não relataram quaisquer ideias de se matar. É de relevância citar o item de pessimismo em que um total de 4 idosos (20\%) apresentaram este sintoma enquanto a maioria dos idosos um total de 16 equivalente a (80\%) não são pessimistas.

Através dos dados coletados pode-se observar que nos indicadores de maior relevância para o diagnóstico da depressão de acordo Beck (BDI), os idosos não apresentaram uma porcentagem favorável de respostas ligadas aos indicadores de pessimismo e ideias suicidas o que demonstra que estes no momento da aplicação do instrumento não apresentaram indicativos de um eventual suicídio e poucos pensamentos pessimistas.

\section{Gráfico 1: Prevalência da depressão}

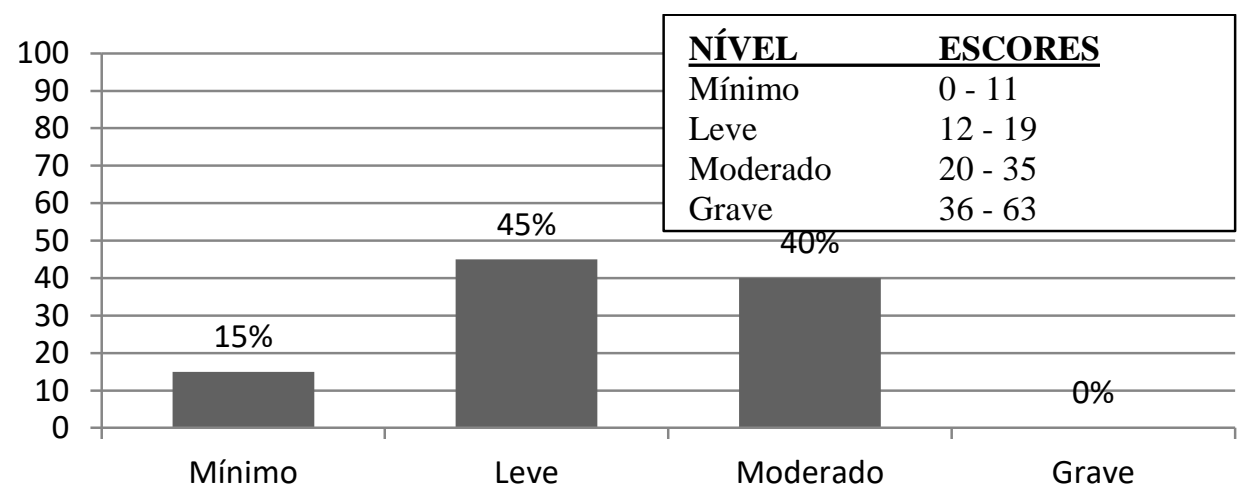


A pesquisa foi composta por uma amostra de 20 idosos equivalente $(100 \%)$ dos indivíduos que participam assiduamente do Projeto de Extensão "Qualidade de Vida na Terceira Idade" no CRAS Vila Nova - Gurupi-TO, a coleta deu-se através do Instrumento BDI em que tem por finalidade averiguar a prevalência e os níveis de depressão.

Diante do exposto, a prevalência da depressão nos idosos participantes da pesquisa, um total de 9 (nove) idosos equivalente a (45\%) apresentaram depressão leve, 8 idosos equivalente a (40\%) apresentaram depressão moderada e 3 idosos equivalente a (15\%) apresentaram depressão mínima. Com os dados demonstrados através do gráfico verificou-se, que não há indicativos de depressão grave nos participantes do Projeto de Extensão Qualidade de Vida na terceira idade do CRAS- Vila Nova em Gurupi-To, sendo importante o acompanhamento dos casos de depressão moderada para que não evolua para quadros mais graves.

Desse montante de acordo exposto, os indivíduos que apresentaram depressão moderada um total de 8 (oito) idosos equivalente a (40\%) que foi o nível mais alto averiguado com a pesquisa, vale ressaltar que com relação ao sexo um total de 6 idosos (30)\% da amostra foram do sexo feminino e um total 2 (dois) idosos (10\%) do sexo masculino, com relação a escolaridade foi possível identificar que um total 3 idosos (15\%) são analfabetos, 4 (quatro) idosos (20\%) tem ensino fundamental incompleto e 1 idoso $(5 \%)$ tem ensino médico completo.

Diante do tópico estado civil pode-se averiguar que a maioria dos idosos que apresentaram depressão moderada um total de 7 idosos (35\%) são viúvos e um idoso (5\%) é casado. Diante dos dados adquiridos com relação à ocupação um total de 4 idosos (20\%) são aposentados e 4 idosos (20\%) do lar. Com o tópico relacionado à idade podese identificar que 4 idosos (20\%) tem idade de 60 a 70 anos, 2 idosos (10\%) tem idade de 70 a 80 anos e 2 idosos (10\%) com idade entre 80 a 90 anos. Desse modo foi possível verificar que os dados expressivos na maioria dos idosos que apresentaram depressão moderada, é que a maior parte é do sexo feminino, viúvos e nos primeiros 10 anos da velhice.

\section{Gráfico 2: Sexo}




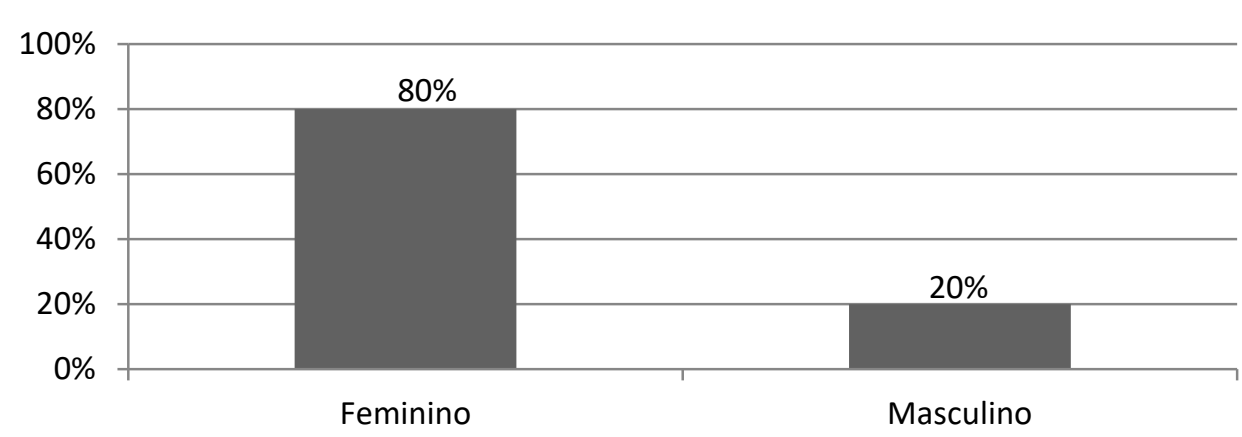

Conforme o (gráfico 2) que se refere ao sexo houve um número significativo de idosos do sexo feminino comparado aos participantes do sexo masculino. Sendo que, um total de 16 idosos equivalente a (80\%) dos indivíduos que participam assiduamente do Projeto de Extensão Qualidade de vida na Terceira Idade no CRAS - Vila Nova são mulheres e um total de 4 idosos equivalente a (20\%) dos participantes são homens.

\section{Gráfico 3: Escolaridade}

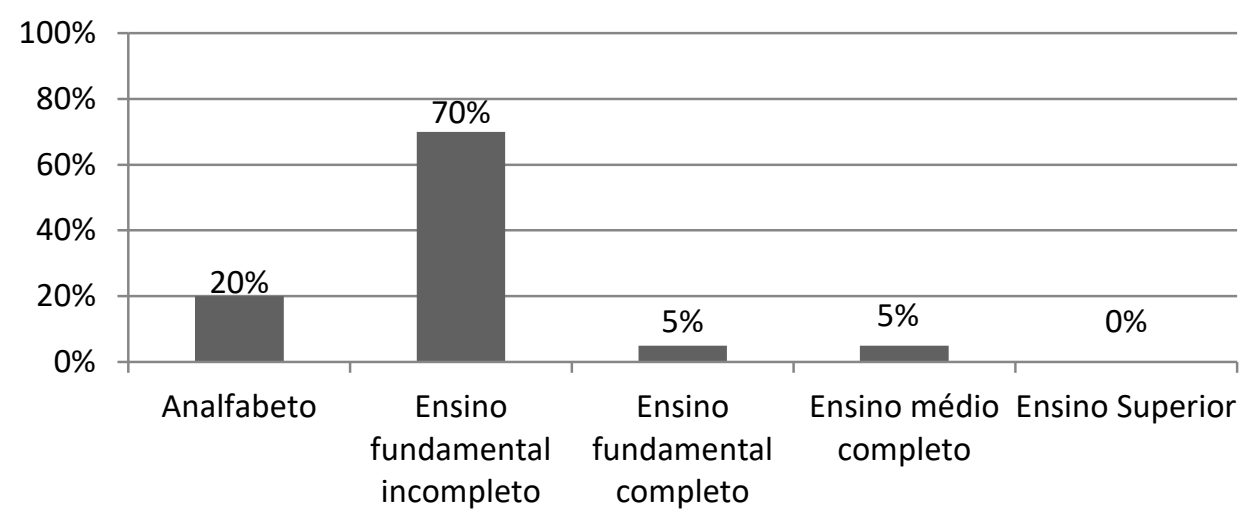

De acordo o (gráfico 3) que se refere escolaridade verificou-se que, um total de 14 idosos equivalente a $(70 \%)$ tem o ensino fundamental incompleto e 4 idosos equivalente a (20\%) são analfabetos. Com a pesquisa foi possível observar que nenhum dos 20 idosos (100\%) tem ensino superior.

\section{Gráfico 4: Estado civil}




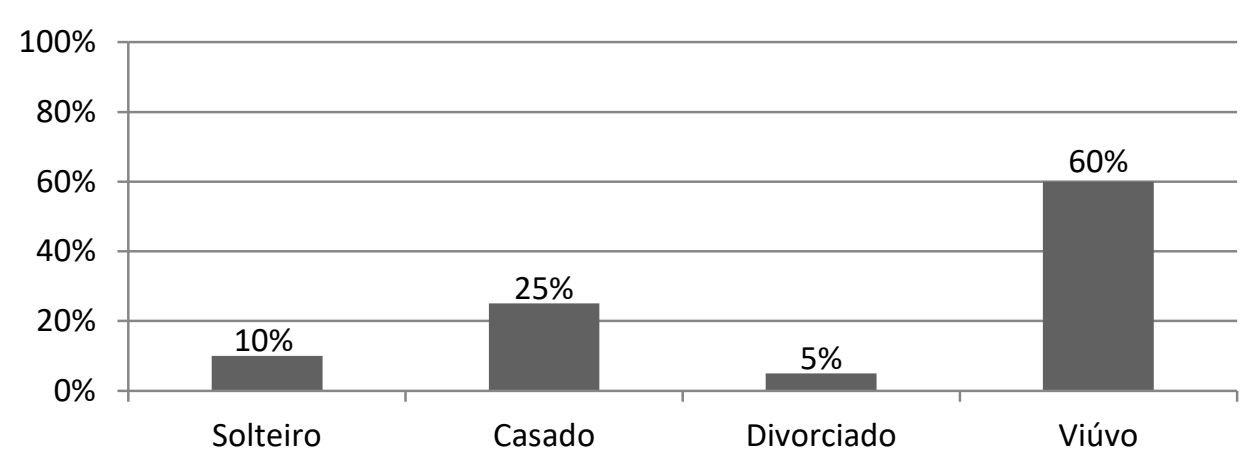

Conforme o gráfico acima referente ao estado civil, observou-se que a maioria dos participantes da pesquisa são viúvos e casados. Sendo que um total de 12 idosos equivalente a (60\%) são viúvos, e 5 idosos equivalente a (25\%) são casados. Ressaltando que o menor percentual relacionado ao estado civil, foi verificado que apenas um idoso equivalente a (5\%) é divorciado.

\section{Gráfico 5: Ocupação}

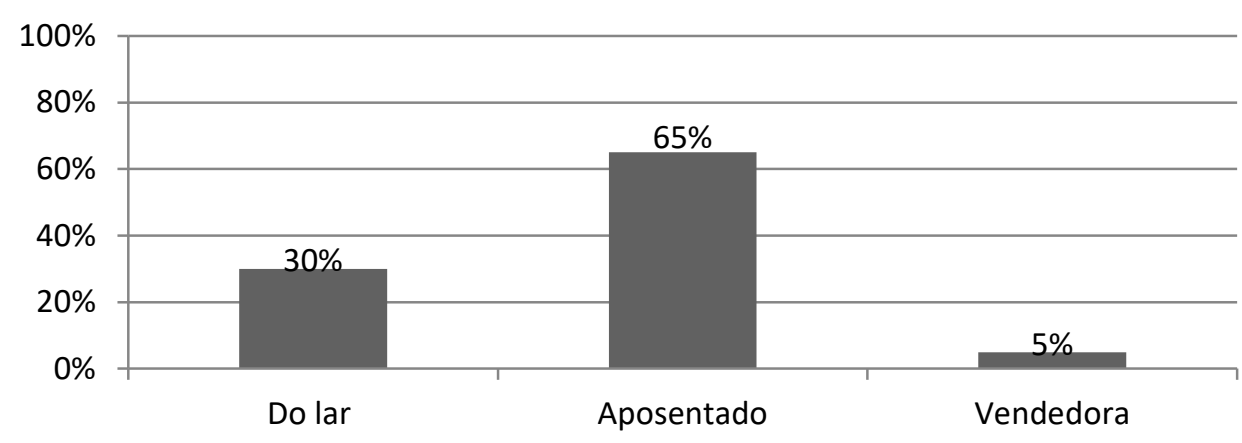

O gráfico acima refere-se à ocupação, observa-se que um total de 13 idosos equivalente a (65\%) são aposentados, 6 idosos equivalente a (30\%) são do lar e um idoso (5\%) ocupa o cargo de vendedora.

\section{Gráfico 6: Idade}




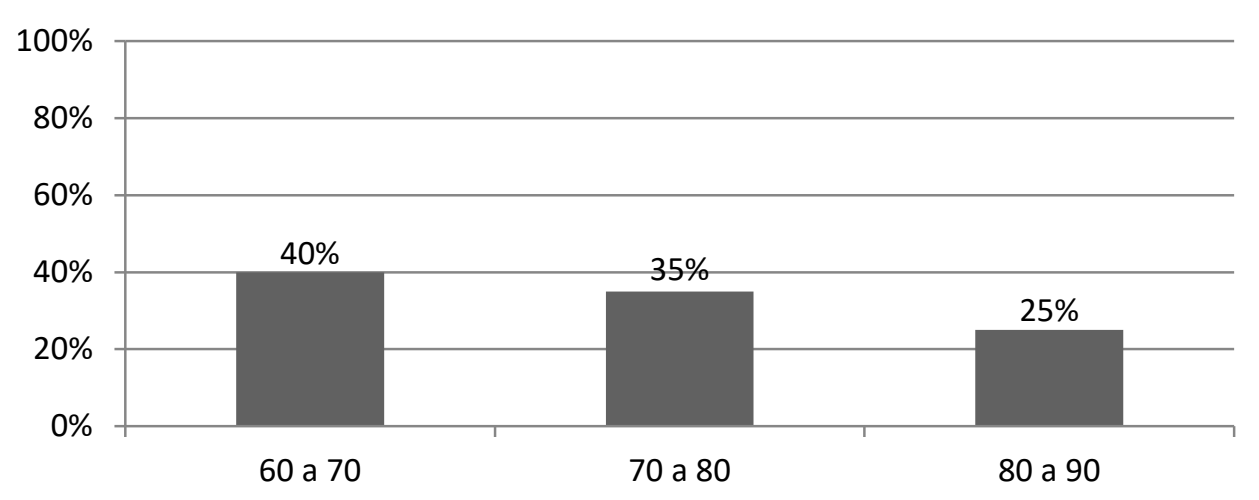

O gráfico acima refere-se à idade, onde demonstra que a maioria dos participantes da pesquisa, um total de 8 idosos equivalente a (40\%) tem idade entre 60 a 70 anos. 0 menor percentual indica um total de 5 idosos equivalente a (25\%) os quais tem idade entre 80 a 90 anos.

Os autores Borges e Dalmolin (2012), evidenciam que os principais fatores contribuintes para a depressão em idosos são: solidão, viuvez, divórcio, baixo nível de instrução e situação socioeconômico dentre outros. Diante do exposto com a pesquisa foi possível verificar idosos que apresentaram depressão moderada. Com os dados coletados com o BDI, foram confirmados que, a depressão está relacionada à solidão, viuvez, baixo nível instrução e situação socioeconômico.

Com base nos resultados apresentados os autores Melo e Teixeira (2011) destacam que com o chegar da idade os indivíduos vão perdendo sua vitalidade e suas forças vão diminuindo, através da perda das atividades em decorrência do envelhecer, o idoso sente-se cada vez mais dependente e menos ativo, com isso as principais consequências são: redução da rede social, problemas conjugais, perda do poder aquisitivo e a vulnerabilidade às diversas doenças.

Diante do discurso do autor destaca-se que, na pesquisa os participantes do Projeto de Extensão "Qualidade de Vida na terceira idade", apresentam as consequências do envelhecer em decorrência da idade, porém apesar das limitações estes conseguem desenvolver atividades e aparentemente sentem-se motivados em realizar trabalhos em grupo o que confirma com a presença assídua dos participantes.

Deste modo, os grupos terapêuticos com idosos depressivos têm mostrado eficácia, pois através destes os idosos veem a oportunidade de compartilhar experiências, angústias, desejos de uma melhor qualidade de vida. Esses grupos terapêuticos com idosos têm a finalidade de socializar os mesmos, proporcionando aumento da autoestima 
e resolução de conflitos intrapsíquicos e interpessoais (CARVALHO e FERNANDES, 2002).

\section{CONCLUSÃO/CONSIDERAÇÕES FINAIS}

Considerando os resultados obtidos a partir dos dados coletados por meio deste trabalho, que os objetivos foram alcançados.

Tendo em vista que a depressão é uma doença comum entre idosos e necessita de conhecimentos científicos com o público alvo, favorecendo e contribuindo positivamente para que os profissionais da instituição possam intervir na melhoria da qualidade de vida dos idosos.

Portanto, é notável a importância do profissional da saúde nas equipes deste tipo de grupo, pois o mesmo deverá estar atento para perceber sintomas depressivos, e assim encaminhar o indivíduo para um acompanhamento médico e psicológico quando verificar evidência de depressão.

Assim, foi possível confirmar que o trabalho do Projeto de Extensão Qualidade de Vida na terceira idade do CRAS - Vila Nova é de grande relevância, pois diante da amostra de 20 idosos equivalentes a (100\%), a maioria destes apresentaram depressão leve e não houve indicadores de depressão grave. O que nos remete que as participações destes idosos no grupo podem estar trazendo benefícios para uma melhor qualidade de vida na terceira idade.

A pesquisa beneficiou a instituição obtendo dados científicos acerca do público alvo, a sociedade por ter uma pesquisa específica com o público idoso, a instituição Centro Universitário Unirg com o Projeto de Extensão "Qualidade de Vida na Terceira Idade" da própria instituição e os idosos participantes do projeto para tomarem consciência de sua saúde mental.

Portanto, sugere-se como possíveis intervenções a este público que os mesmos continuem participando do projeto de Extensão qualidade de vida, tendo em vista que não apresentaram depressão grave. O grupo pode manter e prevenir sua saúde mental, bem como preservar sua rede de relacionamentos sociais. A participação dos mesmos em atividades grupais que envolvam dinâmicas de grupo, com atividades voltadas a temática depressão, pode ser importante para sua qualidade de vida.

\section{AGRADECIMENTOS}


Agradecemos á Instituição CRAS Vila Nova pelo ambiente criativo e amigável que nos proporcionou desenvolver a pesquisa e a instituição Centro Universitário Unirg por ter dado a oportunidade de obter dados científicos do Projeto "Qualidade de Vida na Terceira Idade" da própria instituição.

\section{REFERÊNCIAS}

ARAUJO, E. N . P. Práticas Psicogerontológicas nos Cuidados de Idosos. Curitiba: Juruá, p.144, 2012.

BRASIL. Estatuto do Idoso, Dispositivos Constitucionais Pertinentes Lei no 10.741, de 1은 de outubro de 2003. Normas correlatas índice temático

BRASIL. Ministério do Desenvolvimento Social (MDS) disponível em: www.mds.com.br. Acesso em 06 de novembro de 2015.

BRASIL. Resolução do Conselho Federal de Psicologia.CFP no 002/2003.

BECK, A. T. e STEER, R. A. Beck Depression Inventory. Manual. San Antônio: Psychology Corporation. 1993

BORGES, D. T. e DALMOLIN, B. M. Depressão em Idosos de uma Comunidade assistida pela Estratégia de Saúde da Família em Passos Fundo - RS. Rev. Bras. Med. Farm. Comunidade Florianópolis, v.7, n.23, p.75-82, abr-jun, 2012.

CARVALHO V. F. C. e FERNANDES M. E. D. Depressão no Idoso. In: NETTO, Matheus Papaléo. Gerontologia: a Velhice e o Envelhecimento em Visão Globalizada. São Paulo: Atheneu, 2002. P. 160-173.

CUNHA, J. A. Manual daversão em português das Escalas de Beck. São Paulo. Casa do Psicólogo, Pearson, 2011.

GARBIN, et al. O Envelhecimento na Perspectiva do Cuidador de Idosos. Ciência e Saúde Coletiva, v. 15, n.6, p. 2941-2948, 2010.

GIL, A. C. Como elaborar projetos de pesquisa. 4. Ed. São Paulo: Atlas, 2008.

MELlO E. e TEIXEIRA, M.B. Depressão em Idosos. Rev. Saúde, v.5, n.1, p. 42-53, 2011.

PARADELA, E. M. P. Depressão em Idosos. Revista Hospital Universitário Pedro Ernesto. Ano 10, v. 5, n. 1, 2011.

SILVA, E. R., et al. Prevalência e Fatores Associados à Depressão entre Idosos institucionalizados: subsídio ao cuidado de enfermagem. Rev. Esc. Enferm. USP, v. 46, n. 6, p. 1387-93, 2012.

SILVA, B. T. e SANTOS. S.S.C. Cuidados aos idosos institucionalizados - opiniões do sujeito coletivo enfermeiropara 2026. Acta Paul Enferm. V. 23, n.6, p. 775-81, 2010. 